\title{
Answer To Test Yourself Question: Right medial hip pain
}

\author{
E. Smith ${ }^{1} \cdot$ R. Rajakulasingam ${ }^{2} \cdot$ A. M. Davies ${ }^{1} \cdot$ S. L. James ${ }^{1} \cdot$ R. Botchu ${ }^{1}$
}

Received: 23 October 2020 / Revised: 23 October 2020 / Accepted: 15 November 2020 / Published online: 25 November 2020

(C) ISS 2020

\section{Diagnosis}

Lesser trochanter avulsion fracture secondary to underlying intra-osseous haemangioma.

\section{Discussion}

AP pelvic radiograph (Fig. 1) demonstrates avulsion of the right lesser trochanter with subtle sclerosis along the medial border of the proximal femoral shaft. Given the patient's age and absence of significant trauma, a secondary metastatic deposit was the main differential diagnosis. MRI demonstrated florid low T1 and high STIR signal at the site of avulsion (Figs. 2a and b). Medially, there was predominantly fatty signal with linear low $\mathrm{T} 1$ and heterogeneous STIR signal striations in the adjacent femoral shaft, suspicious for an intraosseous haemangioma (IOH). Although not overtly malignant, the unusual imaging findings warranted CT guided biopsy of the avulsed lesser trochanter. Prebiopsy planning CT scan (Figs. 3a and b) showed coarse trabeculation throughout the medullary cavity and cortex of the proximal femoral shaft. The described changes were most pronounced just medial to the avulsed lesser trochanter. The absence of an aggressive periosteal reaction, lytic area and wide zone of transition with normal appearing bone distally favoured a benign underlying process. Histology (Fig. 4) revealed vascular proliferation lined by a single layer of endothelial cells in keeping with an IOH. The patient was treated conservatively.

The case presentation can be found at doi: 10.1007/s00256-020-03678-4

R. Botchu

drbrajesh@yahoo.com

1 Department of Musculoskeletal Imaging, Royal Orthopaedic Hospital, Birmingham, UK

2 Department of Musculoskeletal Imaging, Royal National Orthopaedic Hospital, Stanmore, UK
Isolated lesser trochanteric fractures in adults are considered a metastatic deposit unless proven otherwise [1]. Primary bone tumours affecting the lesser trochanter are very rare but include osteochondroma and fibrous dysplasia [2]. The lack of a cartilage cap/stalk base went against osteochondroma and the absence of a ground glass appearance and atypical MRI signal characteristics went against fibrous dysplasia. IOH of the proximal femur is very rare, with a literature search revealing 5 cases reports, with only one involving the lesser trochanter with pathological fracture [3]. IOH is usually found in the axial skeleton and when found in the appendicular skeleton are usually in the scapula, ribs, clavicle and pelvis [4].

$\mathrm{IOH}$ has a variety of radiological appearances, and therefore preoperative imaging diagnosis can be difficult. On radiographs, appearances vary from mild cortical sclerosis as in our case to a lytic, soap-bubble or even coarse loculated appearance [5]. Unlike the majority of aggressive primary bone sarcomas and bony metastases, an expansile osteolytic appearance is their least common presentation [5]. MRI appearances can also vary with low, intermediate or high T1 signal. They are usually hyperintense on T2 and STIR sequences owing to free water in stagnant blood within the haemangioma [5]. CT typically shows coarse bony trabeculation and matrix mineralisation [4].

The histopathological diagnosis of $\mathrm{IOH}$ following bone biopsy or curettage can be challenging and should be reported by experienced pathologists. The aforementioned procedures typically disrupt the thin-walled blood vessels resulting in histological sections of just blood clot with scattered bone trabeculae. Operative treatment ranges from curettage and bone grafting with placement of an internal fixation device or even reconstruction with a hip endoprosthesis [6].

Isolated non-traumatic avulsion fractures of the lesser trochanter in adults are rare, almost exclusively occurring in metastatic neoplasms. However, rare benign causes such as IOH need to be considered when presented with unusual imaging findings.

\section{Compliance with ethical standards}

Conflicts of interest None 
Fig. 3 a Coronal CT image of the right hip demonstrates coarse trabeculation throughout the right proximal femur with sclerosis along the medial border. The lesser trochanter is avulsed. Note the relatively sharp demarcation between this and normal bone matrix more distally (arrow). b CT biopsy showing the needle tip within the avulsed lesser trochanter and adjacent soft tissues

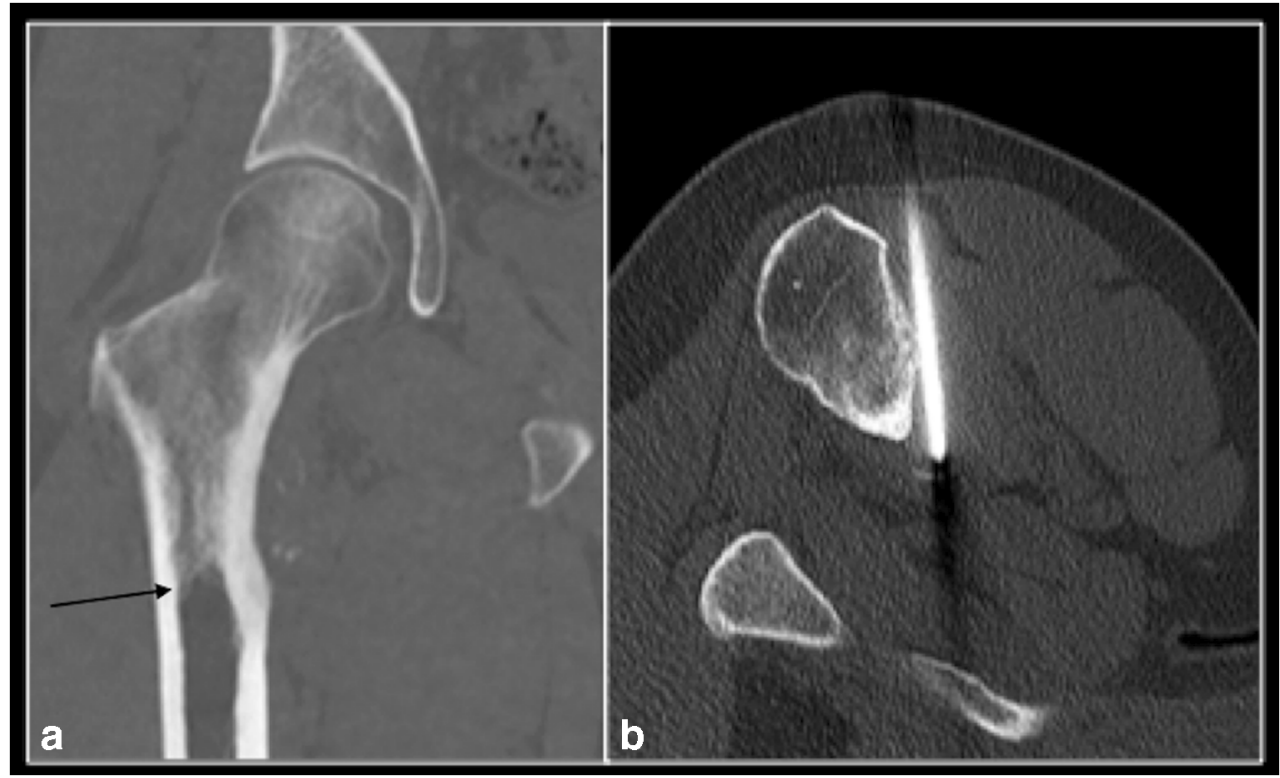

Fig. 4 Microphotograph (H\&E x 20 magnification) shows a collection of capillaries and small to medium thick-walled vascular channels consistent with haemangioma

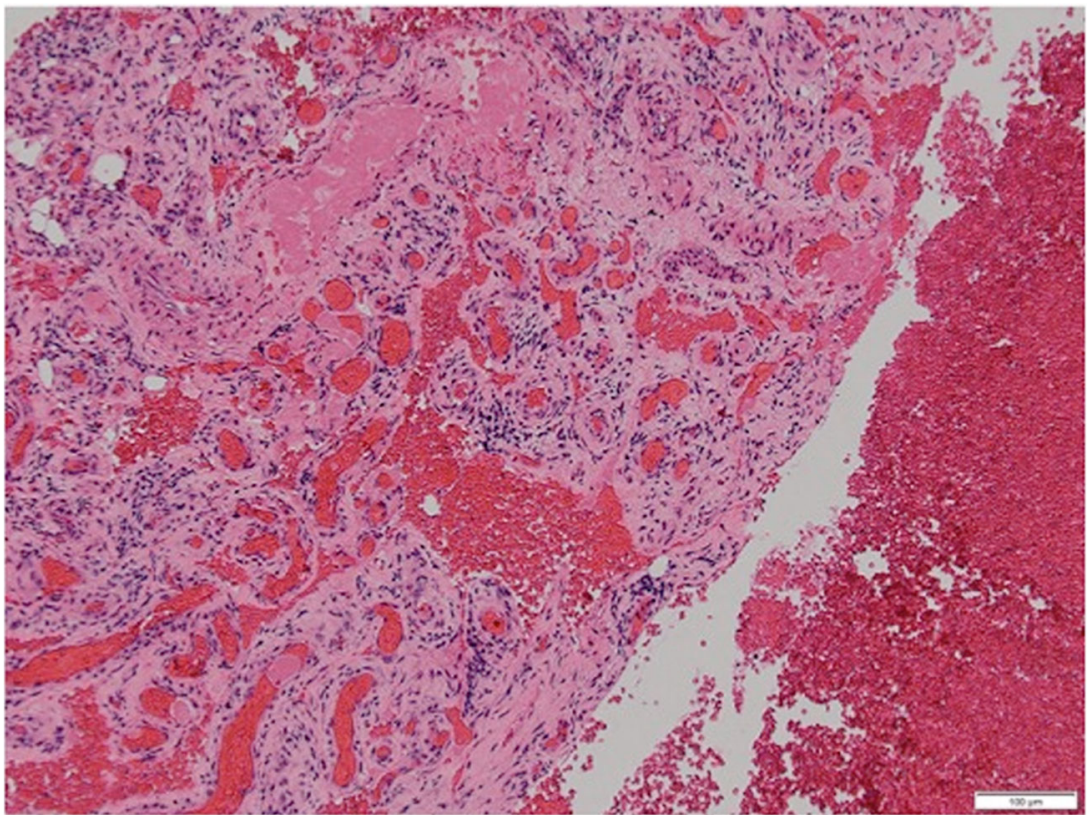

Financial disclosures. None

\section{References}

1. Saad A, Hanif U, Evans S, Iqbal A, Davies M, James S, et al. Isolated primary bone tumours of the lesser trochanter: demographics, diagnosis and management. J Clin Orthop Trauma. 2019;10(6):1046-9.

2. James SL, Davies AM. Atraumatic avulsion of the lesser trochanter as an indicator of tumour infiltration. Eur Radiol. 2006;16:512-4.

3. Aldamegh MS, Alolayan MA, Almukaibil NF. Intraosseous haemangiomas of the proximal femur: a case report. IAJPS. 2018;05(12):15791-5.
4. Rigopoulou A, Saifuddin A. Intraosseous hemangioma of the appendicular skeleton: imaging features of 15 cases, and a review of the literature. Skeletal Radiol. 2012;41:1525-36.

5. Chawla A, Singrakhia M, Maheshwari M, Modi N, Parmar H. Intraosseous haemangioma of the proximal femur: imaging findings. Br J Radiol. 2006;79(944):e64-6.

6. Shih HN, Cheng CY, Chen YJ, Huang TJ, Hsu RW. Treatment of the femoral neck amd trochanteric benign lesions. Clin Orthop Relat Res. 1996:220-6.

Publisher's note Springer Nature remains neutral with regard to jurisdictional claims in published maps and institutional affiliations. 